\title{
Research on Urban Logistics Infrastructure: An Empirical Study of China
}

\author{
Changbing Jiang, Daqiang Chen
}

College of Computer and Information Management, Zhejiang Gongshang University, Hangzhou, China.

Email: johncabin@mail.zjgsu.edu.cn,chendaqiang@mail.zjgsu.edu.cn

Received February $26^{\text {th }}, 2009$; March $26^{\text {th }}, 2009$; accepted April $20^{\text {th }}, 2009$.

\begin{abstract}
Urban Logistics Infrastructure (ULI) is an important area of urban competition capability. The connotation of Urban Logistics Capability (ULC) is analyzed in this paper. Compared with the extensive research on ULC in developed world, empirical work is still rare in China. In this paper the theory of ULC is firstly overviewed. Then a new evaluation index system for ULC evaluation is set up which contains factors that reflect the market supply and demand, economic development and transportation accessibility. Secondly, an empirical study is carried out by using Hierarchical Cluster Analysis (HCA) and Principal Component Analysis (PCA) method to classify ULC into 3 clusters for 30 cities in People's Republic of China. Thirdly, according to the characteristics of the 3 clusters, suggestions are proposed for improving their ULI. Finally, after comparing different ULC of 30 cities in People's Republic of China, this paper focuses on that different logistics infrastructure including Hub, Central Distribution Center \& Cross Docking Center, Regional Distribution Center or Distribution Center should be build reasonably in order to meet the customer's requirement in the 3 different cluster cities.
\end{abstract}

Keywords: urban logistics, performance, hierarchical cluster analysis, principal component analysis

\section{Introduction}

With the accession into the WTO, modern logistics in China possess the great development opportunity [1]. Shanghai, Hong Kong and Guangzhou, some of the major gates to the outside world in China, plan to build themselves into major international logistics center in 5 to 10 years [2]. Therefore the research of Urban Logistics Infrastructure (ULI) has recently become a hot topic in the logistics area.

Urban Logistics Infrastructure (ULI) including Logistics Hub, Central Distribution Center, Cross Docking Center, Regional Distribution Center and Distribution Center is an important area of Urban Logistics Capability (ULC) [3]. Mentzer and Konrad reviewed urban logistics performance measurement practices from an efficiency and effectiveness perspective [4]. Much more attention is paid to freight transport on an interurban level, due to the evolution of supply chain analysis, but this attention is basically devoted to cost factors, which are to be minimized in order to improve the efficiency of the urban logistics system. However, ULI which is an important component of ULC should be re-engineered in order to improve the effectiveness of the urban logistics system [5]. From 1952 to 2003, the large-scale city has increased from 9 to 49 . Therefore, we need to construct different ULI according to each city's logistics capability.

This paper is organized into 5 sections. In Section 1, a brief description of logistics for metropolitan cities in China is introduced. Hierarchical Cluster Analysis (HCA) and Principal Component Analysis (PCA) method are explained in Section 2. After comparing and analyzing different evaluation system of ULC and overview of ULC theory, a new ULC evaluation system is proposed in Section 3, which is composed of market supply and demand, economic development and transportation accessibility. In Section 4, ULC is classified into 3 clusters for 30 sample cities in China using Hierarchical Cluster Analysis (HCA) and Principal Component Analysis (PCA) method. In the final section conclusions of the study is summarized, and further research for this study is suggested.

\section{Research Methodologies}

\subsection{The Method of Hierarchical Cluster Analysis}

Clustering is one of the most important and primitive activities of human beings, dating back to Aristotle. 
Given a set of data objects (also known as patterns, entities, instances, observances, or units), cluster analysis aims to explore natural and hidden data structure and to provide insights to the questions such as, "Are there any clusters (groups, subsets, or categories) in the data, and if yes, how many clusters are in the data?" More specifically, supposing we have a set of $\mathrm{N}$ data objects with d features (attributes, dimensions, or variables) $\mathrm{X}=\left\{\mathrm{x}_{1}, \ldots, \mathrm{x}_{j}, \ldots, \mathrm{x}_{N}\right\}$, where $\mathrm{x}_{\mathrm{j}}=\left(\mathrm{x}_{j 1}, \mathrm{x}_{j 2}, \ldots, \mathrm{x}_{j \mathrm{~d}}\right) \in \mathfrak{R}^{d}$, we have the following mathematical descriptions of two types of clustering [6]:

1) Hard partitional clustering attempts to seek a K-partition of X, $C=\left\{C_{1}, \ldots, C_{K}\right\} \quad(K \leq N)$, such that:

$$
\begin{aligned}
& C_{i} \neq \phi, \quad i=1, \cdots K \\
& Y_{i=1}^{K} C_{i}=\mathrm{X} ; \\
& C_{i} \cap C_{j}=\phi, \quad i, j=1, \cdots K, \quad i \neq j .
\end{aligned}
$$

2) Hierarchical clustering attempts to construct a treelike nested structure partition of $\mathrm{X}, H=\left\{H_{1}, \ldots, H_{Q}\right\}$ $(Q \leq N)$, such that $C_{i} \in H_{m}, C_{j} \in H_{l}$, and $\mathrm{m}>1$ imply $C_{i} \subset C_{j}$ or $C_{i} \cap C_{j}=\phi$ for all $i, j \neq i, m, l=1, \ldots, Q$.

As aforementioned, clustering is generally classified as partitional clustering or hierarchical clustering based on the properties of clusters generated [7]. Partitional clustering directly partitions data objects into some pre-specified number of clusters, while hierarchical clustering groups data with a sequence of nested partitions, either from singleton clusters to a cluster including all individuals or vice versa. The former is known as agglomerative hierarchical clustering, and the latter is called divisive hierarchical clustering. As the binary division of data is computationally expensive, we will focus on agglomerative hierarchical clustering, which is more commonly used in practice.

Agglomerative hierarchical clustering generates a result, which is depicted by a binary tree or dendrogram, based on the proximity matrix. The root node of the dendrogram represents the entire data set, and each leaf node is regarded as a data object. The intermediate nodes thus describe the extent to which the objects are proximal to each other, and the height of the dendrogram usually expresses the distance between each pair of data objects or clusters, or a data object and a cluster. The ultimate clustering results can be obtained by cutting the dendrogram at different levels. This representation provides very informative descriptions and visualizations for the potential data clustering structures, especially when real hierarchical relations exist in the data.
More specifically, for a data set with N samples, general agglomerative hierarchical clustering can be summarized by the following procedure:

1) start with $\mathrm{N}$ singleton clusters $C_{i}(i=1, \ldots, N)$ and calculate the proximity matrix for these $\mathrm{N}$ clusters;

$2)$ in the proximity matrix, search the minimal distance $D\left(C_{i}, C_{j}\right)=\min D\left(C_{m}, C_{l}\right) \quad(1 \leq m, l, \leq N, m \neq l)$, where $D(\cdot, \cdot)$ is the distance function, and combine cluster $C_{i}$ and $C_{j}$ to form a new cluster $C_{i j}$;

3) update the proximity matrix by computing the distances between the cluster $C_{i j}$ and the other clusters; and

4) repeat steps $2 \sim 3$ until only one cluster remains.

\subsection{The Method of Principal Component Analysis}

The method used to derive the component scores using ten indicators for reflecting Urban Logistics Capability (ULC) is Principal Component Analysis (PCA). PCA transforms the original set of variables into a smaller set of linear combinations that account for most of the variations of the original set. The principal components are extracted so that first principal component denoted by PC(1) accounts for the largest variation in the data.

Let us consider the variables $X_{1}, X_{2}, \ldots, X_{\mathrm{p}}$. A principal component analysis of this set of variables can generate $\mathrm{p}$ new variables, known as the principal components, $P C_{1}, P C_{2}, \ldots, P C_{\mathrm{p}}$. The principal components can be expressed as follows:

$$
\begin{gathered}
P C_{1}=b_{11} X_{1}+\ldots+b_{1 \mathrm{p}} X_{\mathrm{p}}=X \mathrm{~b}_{1} \\
\cdot \\
\dot{\cdot} \\
P C_{\mathrm{p}}=b_{\mathrm{p} 1} X_{1}+\ldots+b_{\mathrm{pp}} X_{\mathrm{p}}=X b_{\mathrm{p}}
\end{gathered}
$$

or, in general,

$$
P C=X b
$$

where $b$ 's are the coefficients for principal component and each column of $b$ contains the coefficients for one principal component. Here, the coefficient for $P C_{1}$ is chosen such that it's variance is the largest, and $P C_{2}$ is chosen to have the second largest variance subject to the condition that $P C_{1}$ and $P C_{2}$ are uncorrelated, and so on. For any principal component, the coefficients of principal components are chosen such that $\sum_{i=1}^{p} b_{i j}^{2}=b_{j}{ }^{\prime} b_{j}=1$. Now, if we consider that the sample variance-covariance matrix of the original variables, $\mathrm{X}$, is $S_{\mathrm{x}}$ then the coefficient vector, $b_{\mathrm{j}}$, can be obtained by solving the following equations:

$$
\left|S_{x}-\lambda I\right| b=0
$$

where $\lambda$ is the vector of characteristic roots and $b$ is a matrix comprising of the characteristic vectors corre- 
sponding to each characteristic root [8]. There may be $p$ characteristic roots, some of which may be zero if there are linear dependence among the original variables, $\mathrm{X}$. It may be noted here that $P C_{1}$ is computed by using the characteristic vector corresponding to the largest characteristic root, $\lambda_{1}$, similarly, $P C_{2}$ is computed by using characteristic vector corresponding to the second largest characteristic root, $\lambda_{2}$, and so on.

It must be stressed that a principal component analysis does not always work in the sense that a large number of original variables are reduced to a small number of transformed variables. Indeed if the original variables are uncorrelated then the analysis does absolutely nothing. The best results are obtained when the variables are correlated, positively or negatively [9]. One merit of PCA is that an increase in the number of variables that one may wish to include for deriving a composite index imposes very little cost on the analysis and one can include many related variables for deriving the principal components [10].

\section{Evaluation System of Urban Logistics Capability}

Lu and Yang identified the key logistics capabilities indicator for international distribution center operators, based on five key logistics capabilities including customer response, innovation, economic scale, flexible operation and logistics knowledge [11]. Zhang researched the theory of the location planning for logistics park and set up a new index system for logistics park performance evaluation [12].

The comprehensive evaluation on Urban Logistics Capability (ULC) needs a synthetic evaluation system that takes factors as much as possible into consideration to release the objective evaluation for different impacts of different factors on selection of the logistics facility location, i.e. the planning of Urban Logistics Infrastructure. In general, we select 10 factors which are grouped and stated as the following:

\subsection{Supply and Demand Capability}

\subsubsection{Overall Economy Level}

Here we adopt Gross Domestic Product (GDP) for this factor. GDP refers to the final products at market prices produced by all resident units in the region during a certain period of time. Generally the higher this factor is in a given region, the more feasible a synthetic logistics facility locates in this region.

\subsubsection{Industry Developing Level}

Here we adopt Number of State-owned and state-holding Enterprises (NSSE) for this factor. NSSE refer to state-owned enterprises plus state-holding enterprises.
State-owned enterprises (originally known as state-run enterprises with ownership by the whole society) are non-corporate economic entities registered in accordance with the Regulation of the People's Republic of China on the Management of Registration of Legal Enterprises, where all assets are owned by the state.

\subsubsection{Retail Market Level}

Here we adopt Consumption Expenditure of Urban Households (CEUH) for this factor. CEUH refers to total expenditure of the sample households for consumption in daily life, including expenditure on eight categories such as food, clothing, household appliances and services, health care and medical services, transport and communications, recreation, education and cultural services, housing, miscellaneous goods and services.

\subsubsection{Foreign Trade Developing Level}

Here we use the Number of Foreign Funded Enterprises (NFFE) for this factor. Foreign trade enterprise is the main participator of logistics out-sourcing service in China, which associates with a huge amount of international logistics service, thus the higher this factor is in a given region, the more feasible a synthetic logistics facility locates in this region.

\subsubsection{Urban Freight Traffic and Turnover Volume Level}

Here we adopt Freight Traffic (FT) for this factor. FT refers to the volume of freight transported with various means within a specific period of time. This indicator reflects the service of the transport industry towards the national economy and people's living conditions, as well as an important indicator used in formulating and monitoring transport production plans and research into the scale and pace of transport development. Freight transport is calculated in tons. Freight transport is calculated in terms of the actual weight of the goods and takes no account of the type of freight and distance of travel. Generally, the huger the volume is in a given region, the more feasible for a synthetic logistics facility locates in this region.

\subsection{Economic Development Capability}

\subsubsection{Average Economy Level}

Here we adopt GDP per capita (GDPpC) for this factor. GDPpC refers to the GDP of the region divided by the population of the region. Generally the higher this factor is in a given region, the more feasible a synthetic logistics facility locates in this region.

\subsubsection{Social Reproduction}

Here we adopt Total Investment in Fixed Assets in the region (TIFA) for this factor. TIFA refers to the volume of activities in construction and purchases of fixed assets and related fees, expressed in monetary terms. It is a 
comprehensive indicator which shows the size, structure and growth of the investment in fixed assets, providing basis for observing the progress of construction projects and evaluating results of investment. Total investment in fixed assets in the whole country includes, by type of ownership, the investment by the state-owned units, collective units, individuals, joint ownership units, shareholding units, as well as investment by businessmen from foreign countries and from Hong Kong, Macao and Taiwan, and by other units.

\subsection{Transportation Development Level}

\subsubsection{Accessibility of Railway}

Here we adopt Railway Density (RWD) for this factor. RWD is computed by Length of Railway (LRW) divided by area of the region. LRW refers to the total length of the trunk line under passenger and freight transportation (including both full operation and temporary operation). The calculation is based on the actual length of the first line even if this line has a full or partial double track or more tracks, excluding double tracks, station sidings, tracks under the charge of stations, branch lines, special-purpose lines and the non-payable connecting lines. The length of railway in operation is an important indicator to show the development of the infrastructure for the railway transport, and also the essential data to calculate volume of passenger freight transport, traffic density and utilization efficiency of the locomotives and carriages. RWD is a better way to indicate the accessibility of given region. Because different level logistics facilities need a excellent transport network to facilitate its logistics service, railway density index is feasible for this purpose, which is calculated by length of railroad lines in service divides the region total land area.

\subsubsection{Accessibility of Roads}

Here we adopt Highway Density (HWD) for this factor. HWD is computed by Length of Highway (LHW) di- vided by area of the region. LHW refers to the length of highway which are built in conformity with the grades specified by the highway engineering standard formulated by the Ministry of Communications, and have been formally checked and accepted by the departments of highway and put into use. HWD is another better way to indicate the accessibility of given region, Because different level logistics facilities need an excellent transport network to facilitate its logistics service: highway network density index is feasible for this purpose, which is calculated by total length of highway network in service divides the region total land area.

\subsubsection{Transportation Capacity}

Here we adopt Possession of Civil Motor Vehicles (PCMV) for this factor. PCMV refer to the total numbers of vehicles that are registered and received vehicles license tags according to the Work Standard for Motor Vehicles Registration formulated by transport management office under department of public security at the end of reference period. They are divided into following categories according to the structure of motor vehicles: passenger vehicles, trucks and others; and private vehicles and vehicles for units use according to ownerships; working vehicles and non-working vehicles according to kind of usage; large passenger vehicles, medium passenger vehicles, small passenger vehicles and mini passenger vehicle, heavy trucks, light-heavy trucks, light trucks and mini trucks according to sizes of vehicles.

Based on above analysis, we may obtain ULC evaluation index system for macro level logistics facility planning, as showed in Table 1.

\section{Classification of ULC}

\subsection{Sample Cities Selection and Data Statistics}

Due to imperfect evaluation index system for logistics

Table 1. Evaluation index system for ULC

\begin{tabular}{|c|c|c|c|}
\hline First-grade factor & Second-grade factor & Indicator & Abbreviation \\
\hline \multirow{5}{*}{$\begin{array}{l}\text { Supply and demand } \\
\text { capability }\end{array}$} & Overall economy level & $X_{1}-$ Gross Domestic Product (billion yuan) & GDP \\
\hline & Industry developing level & $\begin{array}{l}X_{2}-\text { Number of State-owned and State-holding Enter- } \\
\text { prises (unit) }\end{array}$ & NSSE \\
\hline & Retail market level & $\begin{array}{l}X_{3}-\text { Consumption Expenditure of Urban Households } \\
\text { (billion yuan) }\end{array}$ & CEUH \\
\hline & Foreign trade developing level & $X_{4}-$ Number of Foreign Funded Enterprises (unit) & NFFE \\
\hline & $\begin{array}{l}\text { Urban freight traffic and turn- } \\
\text { over volume level }\end{array}$ & $X_{5}-$ Freight Traffic (million tons) & FT \\
\hline \multirow{2}{*}{$\begin{array}{l}\text { Economic develop- } \\
\text { ment capability }\end{array}$} & Average economy level & $X_{6}-$ GDP per Capita (yuan/person) & GDPpC \\
\hline & Social reproduction & $X_{7}-$ Total Investment in Fixed Assets (billion yuan) & TIFA \\
\hline \multirow{3}{*}{$\begin{array}{l}\text { Transportation acces- } \\
\text { sibility level }\end{array}$} & Accessibility of railway & $X_{8}-$ Railway Density $\left(\mathrm{km} / 1,000 \mathrm{~km}^{2}\right)$ & RWD \\
\hline & Accessibility of roads & $X_{9}$-Highways Density $\left(\mathrm{km} / 1,000 \mathrm{~km}^{2}\right)$ & HWD \\
\hline & Transportation capacity & $X_{10}-$ Possession of Civil Motor Vehicles (1,000 unit) & PCMV \\
\hline
\end{tabular}

Source: Stanley E.Fawcett (1997) [13]; David J. Closs, Thomas J. Goldsby and Steven R. Clinton (1997) [14]; Edward A. Morash, Cornelia L.M. Droge, Shawnee K. vichery (1996) [15]; Daniel F.Lynch, Scott B.Keller, John Ozment (2000) [16], arranged by author. 
static in China, and the statistical indicators are inadequate in Urban Statistical Yearbook of China, it is impossible for the sample we selected to contain all the influence indicators, i.e. to take factors from the available statistical data into consideration to covering influence indicators that we discussed as much as possible.

According to the difference inherent attributes of different city and distribution channel, 30 major cities in
China are selected, which exclude Hong Kong, Macao, Taiwan and Lhasa for Statistical Indicator and method difference. 30 major cities include:

(1) North China economic region with Beijing as the center, covering Tianjin, Shijiazhuang, Taiyuan, Hohhot;

(2) Northeast China economic region with Shenyang as the center, covering Changchun, Harbin;

Table 2. Synthetic scores of 30 cities in China

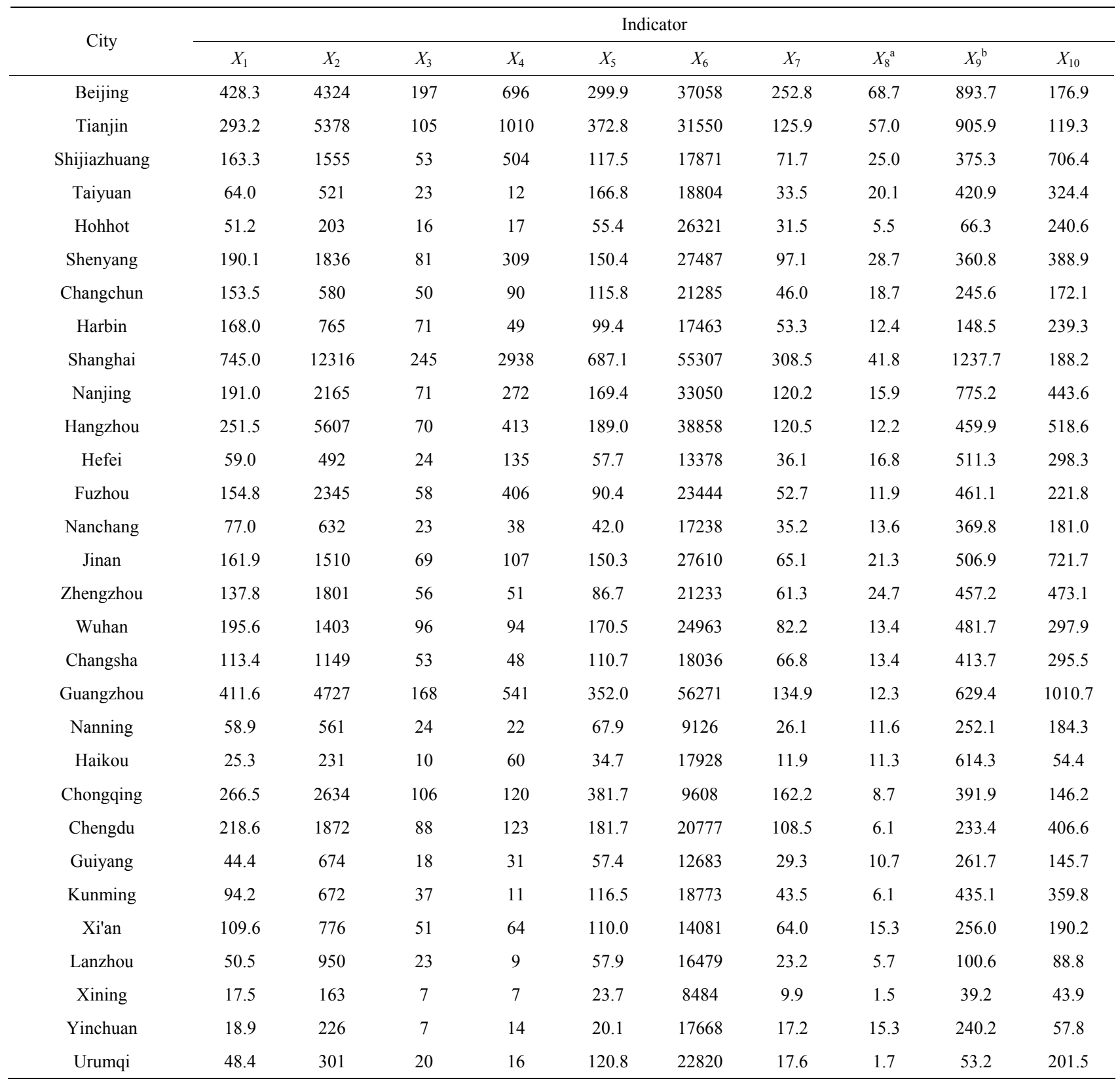

Note: ${ }^{a}$ computed by Total Length of Railways divide by area of the province and, ${ }^{\mathrm{b}}$ computed by Total Length of Highways divide by area of the province.

Source: National Bureau of Statistics of People's Republic of China (2005); China Statistical Yearbook (2005); China City Statistical Yearbook (2005), arranged by author. 
(3) East China economic region with Shanghai as the center, covering Nanjing, Hangzhou, Hefei, Fuzhou, Nanchang, Jinan;

(4) Central China economic region with Guangzhou as the center, covering Zhengzhou, Wuhan, Changsha, Nanning, Haikou;

(5) Southwest China economic region centered in Chongqing, covering Chengdu, Guiyang, Kunming, Lhasa excluded;

(6) Northwest China economic region centered in Xi'an, covering Lanzhou, Xining, Yinchuan, Urumqi.

Based on National Bureau of Statistics of People's Republic of China (2005), China Statistical Yearbook (2005) and China City Statistical Yearbook (2005), ten indicators of the evaluation index system is analyzed above as statistical variables, we have the data showed in Table 2 .

\subsection{Hierarchical Cluster Analysis of ULC}

Hierarchical cluster analysis is a statistical method for finding relatively homogeneous clusters of cases based on measured characteristics. The aim is to maximize between-group variance and to minimize within-group variance. It starts with each case in a separate cluster and then combines the clusters sequentially, reducing the number of clusters at each step until only one cluster is left. In this paper, we apply the Hierarchical cluster method in Statistical Package for Social Sciences to analysis the 30 major cities. The final result is showed in Figure 1.

According to Figure 1, Shanghai and Guangzhou are in one cluster district with rescaled distance cluster combine between 7 and 24, i.e. these two cities have the first cluster with high logistics capacity, and the rest cities are in the other cluster. For rescaled distance cluster combine between 4 and 7 , these 30 cities can be classified into 3 clusters, Shanghai and Guangzhou as the first cluster, Beijing, Hangzhou, Tianjin and Nanjing as the second cluster, and the 24 rest city as the third cluster. And for rescaled distance cluster combine as 3, the third cluster can be classified into 2 clusters, and then the 30 sample city can be classified into 4 clusters.

\subsection{Principal Component Analysis of ULC}

Since most of the indicators suffer from simultaneity and multi-colinearity, Principal Component Analysis (PCA) is best suited for removing such difficulties because it maximizes the variance rather than minimizing the least square distance where any other technique (such as regression analysis) fails to do so.
In this paper, we apply the "Factor Analysis" method in Statistical Package for Social Sciences (SPSS) to analysis the 30 major cities. The results shown in Table 3 suggest a two-factor solution. The eigenvalues clearly show that only two common factors are present by using the criterion of "eigenvalue greater than 1" and it is further confirmed by the fact that the break point occurs at the three eigenvalue of the scree plot (see Figure 2). This being the case, the two-factor solution would appear to be acceptable. Table 4 shows the two Component loadings. From Table 4 we see that RWD and PCMV carried less weight than the others in case of ranking of cities. We also find that the first component explains $73.550 \%$, and the second component explains $11.692 \%$ of the total variation in the data. Since both the eigenvalue of the first component and the second component (in case of ten variables) are greater than 1 , in the present case the two components are used to calculate component score for each city to determine the ranking of selected cities. The two Principal Components (PC) explain about $85.242 \%$ of the variations in the level of ULC. The variables like GDP, NSSE and CEUH played a major role in classifying the cities in terms of ULC compared to the variables such as RWD and PCMV.

In order to calculate the ranking of the selected cities, the principal components can be expressed as follows:

$$
\begin{aligned}
P C_{I}= & 0.362 * X_{1}+0.351 * X_{2}+0.351 * X_{3}+0.332 * X_{4} \\
+ & 0.345 * X_{5}+0.308 * X_{6}+0.35 * X_{7}+0.258 * X_{8} \\
+ & 0.318 * X_{9}+0.093 * X_{10} \\
P C_{2}= & 0.039 * X_{1}-0.032 * X_{2}+0.053 * X_{3}-0.172 * X_{4} \\
& -0.042 * X_{5}+0.34 * X_{6}-0.067 * X_{7}-0.31 * X_{8} \\
& -0.097 * X_{9}+0.859 * X_{10} \\
P C= & 0.317 * X_{1}+0.298 * X_{2}+0.31 * X_{3}+0.263 * X_{4} \\
& +0.292 * X_{5}+0.313 * X_{6}+0.293 * X_{7}+0.18 * X_{8} \\
& +0.261 * X_{9}+0.198 * X_{10}
\end{aligned}
$$

Based on the Hierarchical Cluster Analysis and Principal Component Analysis of ULC mentioned above, we can calculate the ranking of the selected cities. Table 5 shows the ranking of the selected cities based on Principal Component (PC) scores as well as ranking based on GDP. Figure 1 shows the result of the hierarchical cluster analysis.

From Figure 1 and Table 5, We can classify ULC into 3 clusters among the selected cities in People's Republic of China. For the purpose of analysis conveniently, here we define the first cluster as the high LIC cities, the second cluster as the medium ULC cities, the third cluster as the low ULC cities. The high ULC cities including Shanghai, Beijing, Guangzhou and Tianjin, the medium 


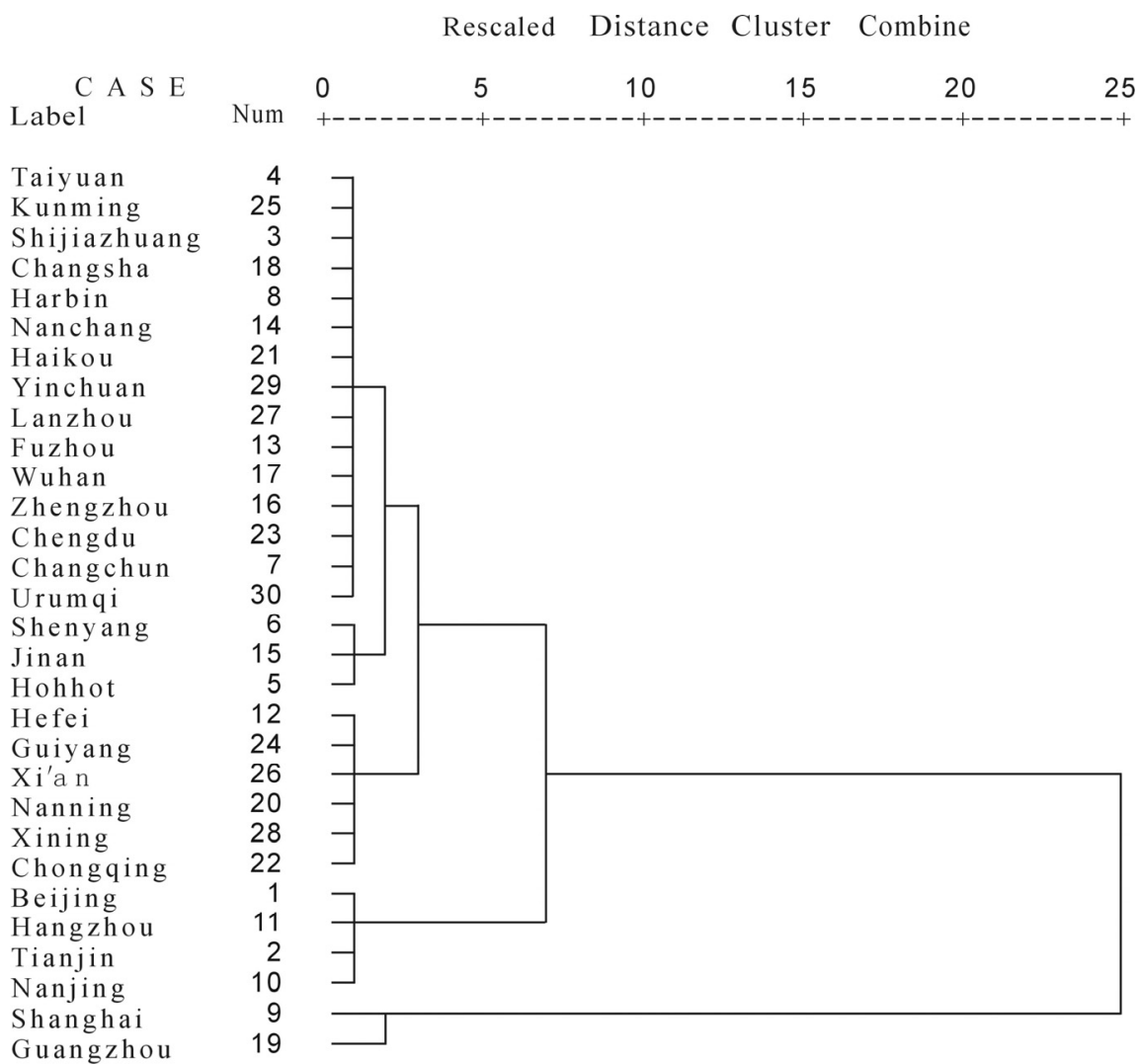

Figure 1. Hierarchical cluster analysis of $\mathbf{3 0}$ major cities

Table 3. Eigenvalues of the correlation matrix (ten variables)

\begin{tabular}{ccccccc}
\hline \multirow{2}{*}{ Component } & \multicolumn{3}{c}{ Initial Eigenvalues } & \multicolumn{3}{c}{ Extraction Sums of Squared Loadings } \\
\cline { 2 - 6 } & Total & \% of Variance & Cumulative \% & Total & \% of Variance & Cumulative \% \\
\hline 1 & 7.355 & 73.550 & 73.550 & 7.355 & 73.550 & 71.692 \\
2 & 1.169 & 11.692 & 85.242 & 1.169 & & 85.242 \\
3 & .608 & 6.082 & 91.324 & & \\
4 & .343 & 3.430 & 94.754 & & \\
5 & .206 & 2.058 & 96.813 & & \\
6 & .159 & 1.590 & 98.403 & & \\
7 & .078 & .785 & 99.187 & & \\
8 & .052 & .517 & 99.705 & & \\
9 & .026 & .257 & 99.962 & & & \\
10 & .004 & .038 & 100.000 & & & \\
\hline
\end{tabular}

Source: Calculated by the author.

ULC cities including Hangzhou, Nanjing, Chongqing, Shenyang, Jinan, Wuhan, Shijiazhuang and Chengdu. the low ULC cities including Zhengzhou, Fuzhou, Changsha, Changchun, Harbin, Taiyuan, Kunming, Xi'an, Hefei, Nanchang, Haikou, Hohhot, Urumqi, Guiyang, Nanning, Lanzhou, Yinchuan and Xining.

From Table 5, we see that, Shanghai topped the list in terms of ULC followed by Beijing and Guangzhou. For the rate of GDP and PC score are quite high, Shanghai is the front runner among the selected cities. The high ULC cities are almost in Bohai Bay region (including Beijing and Tianjin), Yangtze River Delta Region (Shanghai) and Pearl River Delta Region (Guangzhou). The low ULC cities are almost among the Northwest China economic region (including Urumqi, Lanzhou, Yinchuan, Xining, etc.). as illustrated in Table 6 and Figure 3. 
Table 4. Component loadings (Eigenvectors)

\begin{tabular}{ccc}
\hline & \multicolumn{2}{c}{ Component } \\
& $P C_{1}$ & $P C_{2}$ \\
\hline GDP & 0.981 & 0.042 \\
NSSE & 0.952 & -0.035 \\
CEUH & 0.953 & 0.058 \\
NFFE & 0.901 & -0.186 \\
FT & 0.935 & -0.046 \\
GDPpC & 0.836 & 0.367 \\
TIFA & 0.949 & -0.073 \\
RWD & 0.698 & -0.335 \\
HWD & 0.862 & -0.105 \\
PCMV & 0.252 & 0.929 \\
\hline
\end{tabular}

Source: Calculated by the author

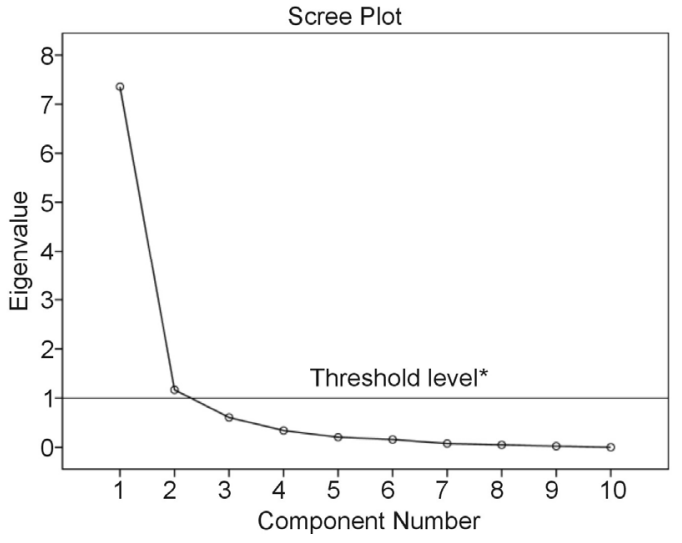

Figure 2. Scree plot of eigenvalues

Note: * Only factors with eigenvalues $>1$ are retained

Table 5. Ranking of 30 cities in China on indicators of environment (using 10 variables)

\begin{tabular}{|c|c|c|c|c|c|c|c|c|}
\hline name & $\begin{array}{c}P C_{1} \\
\text { score }\end{array}$ & $\begin{array}{c}P C_{1} \\
\text { ranking }\end{array}$ & $\begin{array}{c}P C_{2} \\
\text { score }\end{array}$ & $\begin{array}{c}P C_{2} \\
\text { ranking }\end{array}$ & $\begin{array}{c}P C \\
\text { score } \\
\end{array}$ & $\begin{array}{c}P C \\
\text { ranking }\end{array}$ & $G D P$ & $\begin{array}{c}G D P \\
\text { ranking }\end{array}$ \\
\hline Shanghai & 10.209 & 1 & -1.310 & 28 & 8.629 & 1 & 745 & 1 \\
\hline Beijing & 5.041 & 2 & -1.483 & 29 & 4.146 & 2 & 428.3 & 2 \\
\hline Guangzhou & 3.891 & 3 & 3.731 & 1 & 3.869 & 3 & 411.6 & 3 \\
\hline Tianjin & 3.659 & 4 & -1.760 & 30 & 2.916 & 4 & 293.2 & 4 \\
\hline Hangzhou & 1.607 & 5 & 1.311 & 3 & 1.566 & 5 & 251.5 & 6 \\
\hline Nanjing & 1.100 & 6 & 0.736 & 5 & 1.050 & 6 & 191 & 9 \\
\hline Chongqing & 0.886 & 7 & -0.840 & 25 & 0.649 & 7 & 266.5 & 5 \\
\hline Shenyang & 0.536 & 8 & 0.273 & 10 & 0.500 & 8 & 190.1 & 10 \\
\hline Jinan & 0.254 & 9 & 1.765 & 2 & 0.461 & 9 & 161.9 & 13 \\
\hline Wuhan & 0.199 & 10 & 0.212 & 11 & 0.201 & 10 & 195.6 & 8 \\
\hline Shijiazhuang & -0.001 & 11 & 1.258 & 4 & 0.172 & 11 & 163.3 & 12 \\
\hline Chengdu & -0.033 & 12 & 0.712 & 6 & 0.069 & 12 & 218.6 & 7 \\
\hline Zhengzhou & -0.325 & 14 & 0.567 & 7 & -0.203 & 13 & 137.8 & 16 \\
\hline Fuzhou & -0.284 & 13 & -0.194 & 16 & -0.272 & 14 & 154.8 & 14 \\
\hline Changsha & -0.815 & 15 & 0.014 & 13 & -0.701 & 15 & 113.4 & 17 \\
\hline Changchun & -0.960 & 16 & -0.405 & 18 & -0.884 & 16 & 153.5 & 15 \\
\hline Harbin & -1.091 & 17 & -0.055 & 15 & -0.949 & 17 & 168 & 11 \\
\hline Taiyuan & -1.104 & 18 & 0.003 & 14 & -0.952 & 18 & 64 & 21 \\
\hline Kunming & -1.208 & 20 & 0.451 & 8 & -0.980 & 19 & 94.2 & 19 \\
\hline Xi'an & -1.197 & 19 & -0.498 & 20 & -1.101 & 20 & 109.6 & 18 \\
\hline Hefei & -1.400 & 21 & -0.229 & 17 & -1.239 & 21 & 59 & 22 \\
\hline Nanchang & -1.614 & 22 & -0.424 & 19 & -1.451 & 22 & 77 & 20 \\
\hline Haikou & -1.785 & 23 & -0.943 & 27 & -1.670 & 23 & 25.3 & 28 \\
\hline Hohhot & -2.008 & 24 & 0.351 & 9 & -1.685 & 24 & 51.2 & 24 \\
\hline Urumqi & -2.077 & 27 & 0.175 & 12 & -1.769 & 25 & 48.40 & 26 \\
\hline Guiyang & -2.025 & 25 & -0.606 & 23 & -1.830 & 26 & 44.4 & 27 \\
\hline Nanning & -2.038 & 26 & -0.560 & 22 & -1.836 & 27 & 58.9 & 23 \\
\hline Lanzhou & -2.183 & 28 & -0.542 & 21 & -1.958 & 28 & 50.5 & 25 \\
\hline Yinchuan & -2.232 & 29 & -0.877 & 26 & -2.046 & 29 & 18.90 & 29 \\
\hline Xining & -2.999 & 30 & -0.832 & 24 & -2.702 & 30 & 17.50 & 30 \\
\hline
\end{tabular}

Source: Calculated by the author 
Table 6. Profile of LIC

\begin{tabular}{|c|c|c|c|}
\hline Region & $\begin{array}{c}\text { High } \\
P C \geq 2.90\end{array}$ & $\begin{array}{c}\text { Medium } \\
0.00 \leq P C<2.90\end{array}$ & $\begin{array}{c}\text { Low } \\
P C<0.00\end{array}$ \\
\hline North China economic region & Beijing, Tianjin & Shijiazhuang & Taiyuan, Hohhot \\
\hline Northeast China economic region & & Shenyang & Changchun, Harbin \\
\hline East China economic region & Shanghai & $\begin{array}{l}\text { Jinan, Hangzhou, } \\
\text { Nanjing }\end{array}$ & $\begin{array}{l}\text { Fuzhou, Hefei, } \\
\text { Nanchang }\end{array}$ \\
\hline Central China economic region & Guangzhou & Wuhan & $\begin{array}{l}\text { Zhengzhou, Changsha, } \\
\text { Haikou, Nanning }\end{array}$ \\
\hline Southwest China economic region & & Chongqing, Chengdu & Kunming, Guiyang \\
\hline Northwest China economic region & & & $\begin{array}{l}\text { Xi'an, Urumqi, } \\
\text { Lanzhou, Yinchuan, } \\
\text { Xining }\end{array}$ \\
\hline
\end{tabular}

Source: Arranged by the author

The result of classification shows that there are three basic logistics capacity clusters, which is useful for application in China. The between-group variance of the third and fourth cluster is the smallest, i.e. logistics capacities in cities belong to these two clusters are similar to each other and can be modified as one cluster. While the between-group variance of the first, second and third cluster have significant difference, i.e. cities belong to these three clusters, respectively, are different in the logistics capacity.

For the purpose of analysis conveniently, here we define the first cluster city as the Hub/CDC (Hub/ Central Distribution Center \& Cross Docking Center) city, the second cluster as the CDC/RDC (Central Distribution Center \& Cross Docking Center / Regional Distribution Center) and the third cluster as the RDC/DC (Regional Distribution Center / Distribution Center) city, as illustrated in Figure 3.

\section{Urban Logistics Network Structure}

Logistics network is an integration of organizations and facilities within the logistics procedure, and also is an integration network that consists of physical network of product that flows between facilities such as hub, central distribution center, regional distribution center and other kinds of logistics facility, and network of information that flows parallel with the product flow. The aims of urban logistics network structure is to define the location and number of logistics facilities and control the systematic cost with certain service supply level by network analysis and optimal methods.

\subsection{Category of Urban Logistics Facility}

There are four categories urban logistics facility in China, such as hub, central distribution center, regional distribution center and distribution center.

\subsubsection{Hub}

According to DOD in USA, hub refers to an organization that sorts and distributes inbound cargo from wholesale supply sources (airlifted, sealifted, and ground transportable) and/or from within the theater. Suppliers can arrange material and product in hub to supply the large hub or logistics center in service destination by long distance transportation to concentrate the supply, take advantage of common transport and combined loading, improve the logistics active efficiency and productivity, and decrease the procurement and supply cost.

\subsubsection{CDC (Central Distribution Center \& Cross Docking Center)}

Cross Docking Center is the facility where the material or products are received from suppliers, sorted directly to be shipped to a consolidated batch (often including other orders from other suppliers) to the customers by the same vehicle or different without putting them in storage. Its particular advantages reside at the minimization of warehousing and economies of scale in outbound flows (from the distribution center to the customers), and it helps reduce operating costs, increase throughput, reduces inventory levels, and helps in increase of sales space. The material or products handled in CDC are usually of large-size, small-item, and low-frequency.

\subsubsection{RDC (Regional Distribution Center)}

A Regional Distribution Center (RDC) is a collection and consolidation center for finished goods, components and spare parts to be distributed to the distribution center belongs to dealers, importers or other unrelated organizations within or outside the region. Among the functions involved are information network service, repackaging and labeling, and distribution. The material or products handled in CDC are usually of small-size, mul- 
tiple-item, and personality.

\subsubsection{DC (Distribution Center)}

Distribution Centers are foundation of urban logistics network, which usually is a model "warehouse" or other specialized building which is stocked with products to be re-distributed to retailers or wholesalers. In the urban logistics network discussed in this paper, up-level facilities will ship truckloads of products to the distribution center, and then the distribution center will store the product until needed by the retail location and ship the proper quantity to the retails, stores, even the final consumers.

\subsection{Planning of Urban Logistics Facility}

Based on above quantitative analysis of the sample data, and qualitative analysis of four categories urban logistics facility, we locate different facility in different city clusters, and draw up different developing policy respectively.

\subsubsection{Developing Policy for Hub/CDC City}

In $\mathrm{Hub} / \mathrm{CDC}$ city, four tier logistics network is designed, in which goods is transported from the Hub to the CDC, and then distributed to RDC, finally reaches DC, as illustrated in Figure 4.

There is a relatively perfect operation system in $\mathrm{Hub} / \mathrm{CDC}$ city, the focus of urban logistics infrastructure planning is on enhancing the improvement and integration of the logistics systematic function, strengthening the mechanization, automatization and informationization, improving the capacity of handling and efficiency of different logistics facility, developing multimode transportation, facilitating efficient connection between facilities and transport line, optimizing operation process to control the cost.

In practice, advanced operation model should be introduced, i.e. synthetic logistics facility should be built to attract supplier of components and spare parts, and integrated operation system with supply-produce-sale and quick response should be instructed.

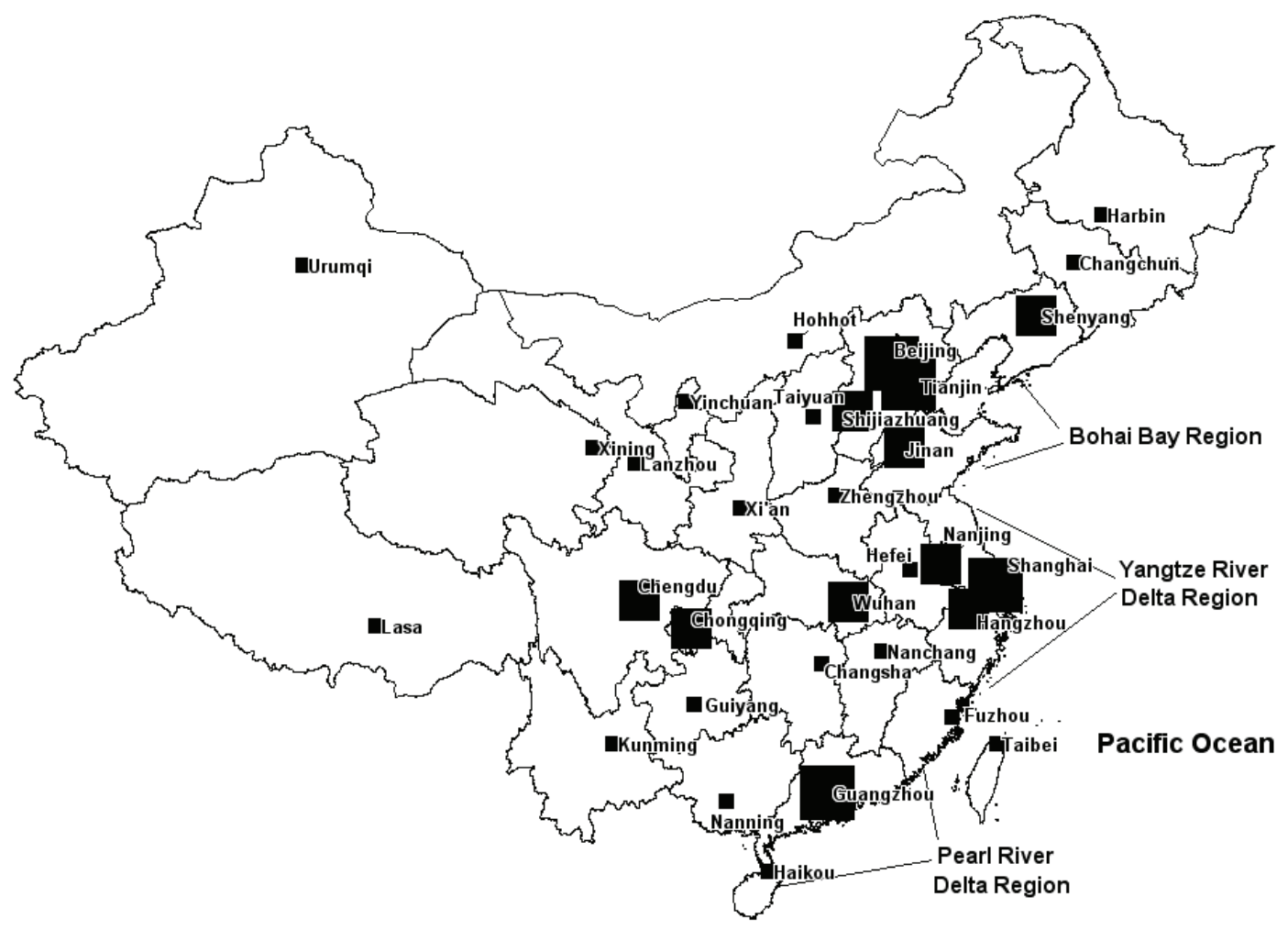

Note:

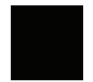

cluster 1: Hub/CDC city, includes 4 cities: Shanghai, Beijing, Guangzhou and Tianjin;

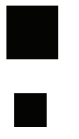

cluster 2: CDC/RDC city, includes 8 cities: Hangzhou, Nanjing, Chongqing, Shenyang, Jinan, Wuhan,

Shijiazhuang and Chengdu;

cluster 3: RDC/DC city, includes 18 cities: Jinan, Hohhot, Harbin, Urumqi, etc.

Figure 3. Classification of $\mathbf{3 0}$ major cities in China according to ULC 


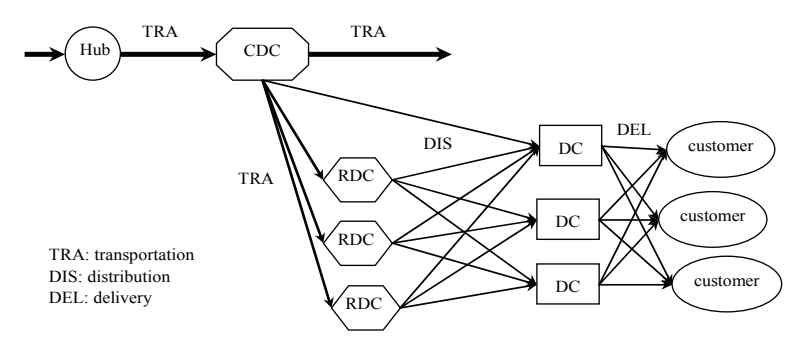

Figure 4. Logistics network for Hub/CDC city

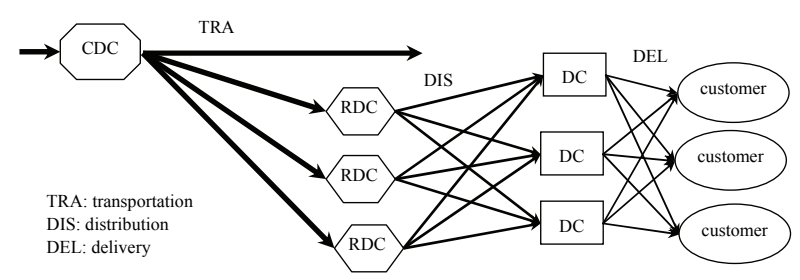

Figure 5. Logistics network for CDC/RDC city

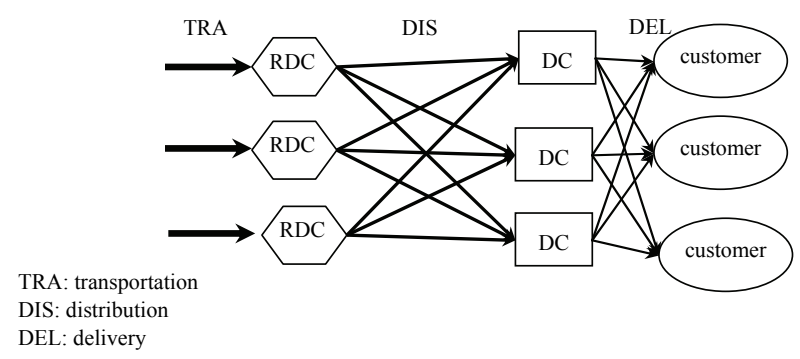

Figure 6. Logistics network for RDC/DC city

\subsubsection{Developing Policy for CDC/RDC City}

In $\mathrm{CDC} / \mathrm{RDC}$ city, three tier logistics network is designed, in which goods is transported from the CDC to the RDC, and then distributed to DC, as illustrated in Figure 5.

In this system, larger RDCs should be built for outboard transport, which should be located around the industry park with convenient transportation network with the surrounding area, i.e. goods or material can distributed to the surrounding area directly through this facility. And lager DC should be built for distributing goods to retails, stores, even the final consumers.

For transportation network constructing, it is the long distance transportation system that should be constructed with urban distribution network improvement as a supplement, i.e. to construct a smooth outboard transport system.

\subsubsection{Developing Policy for RDC/DC City}

In RDC/DC city, two tier logistics network is designed, in which goods is distributed transported from the RDC to the DC, as illustrated in Figure 6.
In this system, DCs should be built to enforce the capacity of small-size, multiple-item, and high-frequency goods order-picking, to improve the capacity of automatic handling, and to quick the response activity. And RDCs should also be built to accept the goods supplied by surrounding area and distribute the goods to DCs timely.

For transportation network constructing, it is the 1 urban distribution network that should be constructed by expansion of traffic road network and traffic capacity improvement, thus a distribution channel, in which the routing optimization within urban area is the focus and long distance transportation is a supplement, should be setup based on urban GIS and freight characters.

\section{Conclusions}

The empirical evidence presented in Section 4 gives a better method of classifying ULC. The following conclusions can be drawn:

(1) 30 sample cities' ULC in China are classified into 3 clusters based on hierarchical cluster analysis method.

(2) We find that China has two Hub/CDC cities including Shanghai and Guangzhou, four CDC/RDC cities including Beijing, Tianjing, Nanjing and Hangzhou, and the other $24 \mathrm{RDC} / \mathrm{DC}$ cities including Jinan, Hohhot, Harbin, Urumqi, etc.

(3) The Hub/CDC and CDC/RDC cities are in coastland area including Bohai Bay region (Beijing), Yangtze River Delta Region (Shanghai, Nanjing and Hangzhou) and Pearl River Delta Region (Guangzhou). The RDC/DC cities are almost among inland area (Changsha, Guiyang, Harbin, Urumqi, etc.).

(4) The evaluation framework of ULC is partly tested by empirical study and needs further and deeper research.

\section{Acknowledgement}

It is a project supported by philosophy and social science in Zhejiang Province (07CGLJ018YBX), the results of Center for Research in Modern Business, Zhejiang Gongshang University (the important research base for high school social and science of High Education Department) and the normal project of philosophy and social science in Hangzhou (D07GL07).

\section{REFERENCES}

[1] M. Goh and C. Ling, "Logistics development in China," International Journal of Physical Distribution \& Logistics Management, 33(10), pp. 886-917, 2003.

[2] Shanghai Information Center, "Logistics to get boost in south China city," People Daily, No. 1, June 5, 2003. 
[3] J. Muñuzuri, J. Larrañeta, L. Onieva, and P. Cortés, "Solutions applicable by local administrations for urban logistics improvement," Cities, 22(1), pp. 15-28, 2005.

[4] Mentzer and Konrad, "An efficiency/effectiveness approach to logistics performance analysis," Journal of Business Logistics, 12(1), pp. 33-61, 1991.

[5] B. Jiang and E. Prater, "Distribution and logistics development in China: The revolution has begun," International Journal of Physical Distribution \& Logistics Management, 32(9), pp. 783-798, 2002.

[6] P. Hansen and B. Jaumard, "Cluster analysis and mathematical programming," Mathematical Programming, 79(1), 191-215, 1997.

[7] R. Xu and D. C. Wunsch II, "Recent advances in cluster analysis," International Journal of Intelligent Computing and Cybernetics, 1(4), 484-508, 2008.

[8] J. R. Harris, "A primer of multivariate statistics (3rd Edition)," Lawrence Erlbaum Associates Publishers Press, 2001.

[9] B. Manly, "Multivariate statistical methods: A primer," Chapman and Hall Press, 1986.

[10] R. Ram, "Composite indices of physical quality of life, basic needs fulfillment and income: A principal compo- nent representation," Journal of Development Economics, 11(1), pp. 227-247, 1982.

[11] C.-S. Lu and C.-C. Yang, "Evaluating key logistics capabilities for international distribution center operators in Taiwan," Transportation Journal, 45(4), pp. 9-27, 2006.

[12] X. D. Zhang, "Research on the theory of the location planning for logistics park," China Logistics Publishing House, Beijing (in Chinese), 2004.

[13] S. E. Fawcett, "Developing a logistics capability to improve the performance of international operations," Journal of Business Logistics, 18(2), pp. 101-127, 1997.

[14] D. J. Closs, T. J. Goldsby, and S. R. Clinton, "Information technology influences on world class logistics capability," International Journal of Physical Distribution \& Logistics Management, 27(1), pp. 4-17, 1997.

[15] E. A. Morash, C. L. M. Droge, and S. K. Vichery, "Strategic logistics capabilities for competitive advantage and firm success," Journal of Business Logistics, 17(1), pp. 1-22, 1996.

[16] D. F. Lynch, S. B. Keller, and J. Ozment, "The effects of logistics capabilities and strategy on firm performance," Journal of Business Logistics, 21(2), pp. 47-67, 2000. 